\title{
Robotic-assisted laparoscopic liver resection in hepatocellular carcinoma
}

\author{
Kevin P. Labadie ${ }^{1}$, James O. Park ${ }^{1,2}$, Jonathan G. Sham ${ }^{1,2}$ \\ 'Department of Surgery, University of Washington, Seattle, WA 98195, USA. \\ ${ }^{2}$ Center for Advanced Minimally Invasive Liver Oncologic Therapies (CAMILOT), University of Washington, Seattle, WA 98195,
} USA.

Correspondence to: Dr. Jonathan G. Sham, Department of Surgery, University of Washington, 1959 Pacific St. NE, Seattle, WA 98195, USA.E-mail: jsham@uw.edu How to cite this article: Labadie KP, Park JO, Sham JG. Robotic-assisted laparoscopic liver resection in hepatocellular carcinoma.
Hepatoma Res 2020;6:65. http://dx.doi.org/10.20517/2394-5079.2020.86

Received: 18 Aug 2020 Accepted: 25 Aug 2020 Published: 12 Sep 2020

Academic Editor: Ho-Seong Han Copy Editor: Cai-Hong Wang Production Editor: Jing Yu

Over the past 20 years the use of laparoscopy has revolutionized hepatic surgery and, in recent years, robotic assistance to the laparoscopic approach has been explored by some centres. Since its approval, there has been a prodigious increase in robotic utilization for surgical operations worldwide ${ }^{[1,2]}$. As its use continues to increase, assessment of efficacy and outcomes is an area of active study.

Preliminary inquiries into robotic-assistance in surgical procedures began in the late 1980 s with a robot designed to perform neurosurgical biopsies with improved precision ${ }^{[3]}$. Innovation accelerated shortly thereafter as multiple governmental organizations, including the United States Army and National Air and Space Administration, became interested in telesurgery - the capability for a surgeon to operate on a physically distanced patient using a robotic interface ${ }^{[4,5]}$. Expertise and technology devised from these efforts later facilitated development of commercial robotic surgical systems for civilian use, including the ROBODOC $^{[6]}$, AESOP system $^{[7]}$, Zeus ${ }^{[8]}$, ARTEMIS $^{[9]}$ and the da Vinci Surgical System ${ }^{[10]}$.

Currently, the most widely used robotic platform worldwide is the da Vinci Surgical system, first approved for use in general laparoscopic surgery by European regulatory agencies in 1999, followed shortly thereafter by the United States Food and Drug Administration in 2000. Initially reserved for smaller operations such as cholecystectomy, the robot is now used for a wide variety of operations ranging from inguinal herniorrhaphy to major hepatectomy. Many surgeons perceive advantages when using robotic systems, which may explain this increased utilization ${ }^{[11]}$. These include wristed articulated instruments, 
3-dimensional high definition optics, improved surgeon ergonomics, diminished surgeon fatigue, as well as a shortened learning curve compared to standard laparoscopic liver surgery. Several disadvantages are emphasized by detractors of the robotic technique, including the need for experienced teams, substantial acquisition and operational costs, and concerns over lack of benefit over standard laparoscopic surgery ${ }^{[12,13]}$.

Robot-assisted laparoscopic liver resection (RALLR) was first described in the $2000 \mathrm{~s}^{[14]}$, with the initial landmark case series of 70 patients reported by Guilianotti and colleagues in $2010^{[15]}$. The indications for RALLR mirror those of the standard laparoscopic approach, including resection of both benign and malignant tumours in patients who can tolerate general anaesthesia and prolonged pneumoperitoneum. Over the last two decades, numerous single and multi-institutional case series have reported outcomes of RALLR in high-volume centres, demonstrating it to be safe, feasible, and effective for minor and major hepatectomies ${ }^{[16]}$. Compared to standard laparoscopic liver resection, perioperative outcomes including operative time and estimated intraoperative blood loss have been shown to be higher in RALLR, but the open conversion rate, Ro resection rate, hospital length of stay, morbidity and mortality are no different than standard laparoscopic hepatectomy ${ }^{[17]}$. Indeed, RALLR recapitulates the main benefits observed with standard laparoscopic hepatectomy in comparison to open hepatectomy, including reduced postoperative complications and shorter length of stay without compromising oncological outcomes such as radical resection rate, overall survival, and disease-free survival rate ${ }^{[18,19]}$. The accumulation of evidence supporting RALLR resulted in international consensus guidelines promoting the development and standardization of robot-assisted laparoscopic hepatectomy in $2018^{[20]}$.

RALLR is indicated for resection of all liver tumour types including hepatocellular carcinoma (HCC). It has been demonstrated to be safely performed for patients with HCC who have well-compensated cirrhosis without signs of severe portal hypertension. Evidence examining the safety and oncological effectiveness of RALLR for HCC is accumulating, with a systematic review published in 2018 highlighting the favourable short-term outcomes reported in over 300 patients from 10 institutional series ${ }^{[21]}$. In the largest series examining RALLR for HCC to date, Chen et al. ${ }^{[22]}$ performed a propensity score matching analysis of 81 RALLR and 81 open liver resections for HCC. In this report, which included approximately $40 \%$ major hepatectomies, operative time was longer in the RALLR compared to open cohort (median of $343 \mathrm{~min} v s$. $220 \mathrm{~min}$ ). However, the intraoperative blood loss (median $282 \mathrm{~mL} v s .263 \mathrm{~mL}$ ), percent of patients requiring an intra-operative blood transfusion (7.4\% vs. 3.7\%), and postoperative complications ( $4.9 \%$ vs. $3.7 \%$ ) were comparable between the two techniques. Notably, the authors observed a significantly reduced length of hospital stay (median 7.5 days vs. 10.1 days), reduced patient-controlled analgesia use on postoperative day 1 and during initial ambulation in the RALLR compared to open hepatectomy cohort. Similar outcomes have been observed in other institutional cohorts; however, it is worth noting that these early published series come from high-volume institutions with extensive experience with laparoscopic liver resection, potentially limiting the generalizability of these findings.

Given the relatively recent adoption of RALLR, studies examining the long-term oncological outcomes in patients after resection for HCC are limited. An early and available outcome that many authors examine as a surrogate for oncological efficacy is the Ro resection rate, as a positive histological margin is known to be associated with a higher incidence of postoperative recurrence in $\mathrm{HCC}^{[23]}$. Ro resection rate may also serve as a hallmark of technical feasibility, as lower Ro resection may indicate suboptimal technical skill or an inherent limitation of the technology (i.e., inability to palpate tumour with minimally invasive approach). Published RALLR Ro resection rates are high, ranging from $85 \%-100 \%$ in most series ${ }^{[16]}$. In regard to longterm oncological outcome data, four of the largest studies examining RALLR for HCC report disease-free survival and overall survival ranging from $72 \%$ to $75 \%$ and $93 \%$ to $98 \%$ at 2 and 3 years postoperatively, respectively ${ }^{[24-26]}$. These mid-term oncological data are encouraging, but will need confirmation with longerterm outcome reporting. 
The modern liver surgeon is afforded with numerous technical approaches to the resection of liver tumours. Although several matched comparisons and meta-analyses have been performed examining the differences between RALLR and several types of laparoscopic liver resection (LLR), many of these are limited by retrospective design and confounded by selection biases ${ }^{[27]}$. One of the largest studies comparing RALLR and LLR matched 57 RALLR to 114 LLR based on background liver disease, extent of resection, diagnosis and other patient demographic factors ${ }^{[28]}$. The authors concluded that RALLR and LLR displayed similar safety and feasibility for hepatectomies, but that more resections approached robotically were completed totally minimally invasively compared to LLR, with a significant proportion of their LLR cohort requiring hand-assist port. The clinical impact of hand-port utilization on postoperative pain and quality of life is unclear. Magistri et al. ${ }^{[29]}$ compared RALLR and LLR specifically for HCC and reported similar outcomes between both approaches.

A specific indication where RALLR may be advantageous to standard LLR is the resection of liver segments in the posterosuperior segments (S1, S4A, S7, S8), which are difficult to approach by standard LLR. Challenges in these approaches for standard LLR include poor visualization, difficulty in haemorrhage control, as well as deep, curved or angled parenchymal transection planes associated with the posterior location of these segments. Although tumourectomies and segmentectomies of these segments can be feasibly approached laparoscopically, they often require expert skill and invasive manoeuvres (i.e., transpleural approach), potentially limiting their generalizability ${ }^{[30,31]}$. Laparoscopic right hepatectomy is often the preferred approached to resections of these lesions since it is technically less challenging. Several studies have evaluated RALLR for parenchymal-sparing resection of lesions in the posterosuperior segments, with authors concluding that RALLR does indeed offer specific advantages to standard LLR and may serve as an alternative to open resection for these lesions ${ }^{[32-36]}$. A review of our institutional series of over 250 RALLR similarly corroborate these findings.

Enhanced efficacy of systemic therapies has provided significant benefit for patients with primary and secondary hepatic malignancies and highlighted the importance of parenchymal sparing resections when performing hepatectomy, as more patients may benefit from resection of intrahepatic recurrence. This trend in management has challenged liver surgeons to totally extirpate tumours while preserving maximal liver parenchyma, which is often damaged following numerous rounds of systemic chemotherapy, to allow for future liver-directed therapies. Performing these resections from a minimally invasive approach may be advantageous to survival by minimizing postoperative complications and potentially expediting a return to adjuvant therapy, although additional confirmatory data are needed. Nevertheless, it is appealing to speculate that performing minimally invasive parenchymal-sparing liver resections is advantageous for certain liver cancer patients.

New technologies to improve pre-operative planning, intra-operative decision making, and surgical training are being developed for the da Vinci robotic surgical system ${ }^{[37]}$. Advances in image-guided liver surgery, surgical resection maps, 3D modelling, and indocyanine green fluorescence with near-infrared fluorescent imaging and $3 \mathrm{D}$ modelling have been developed to assist the surgeon and surgical trainee. In collaboration with Intuitive, our group has helped to develop novel interactive 3D models for preoperative planning and intra-operative navigation [Figure 1]. Numerous three-dimensional virtual-reality robotic surgery simulators now exist for surgical trainees to improve robotic technique and simulate reallife operative situations. Furthermore, development of novel robotic surgical systems by companies such as Medtronic, Johnson \& Johnson, and TransEnterix will only propel ongoing technological innovation. We hope these innovative new technologies translate into improved surgical outcomes for our patients and a well-trained next generation of minimally invasive liver surgeons.

In conclusion, RALLR is a safe and effective approach to the minimally invasive resection of hepatic malignancies. In experienced hands, it is equivalent to the standard laparoscopic approach to anterior 

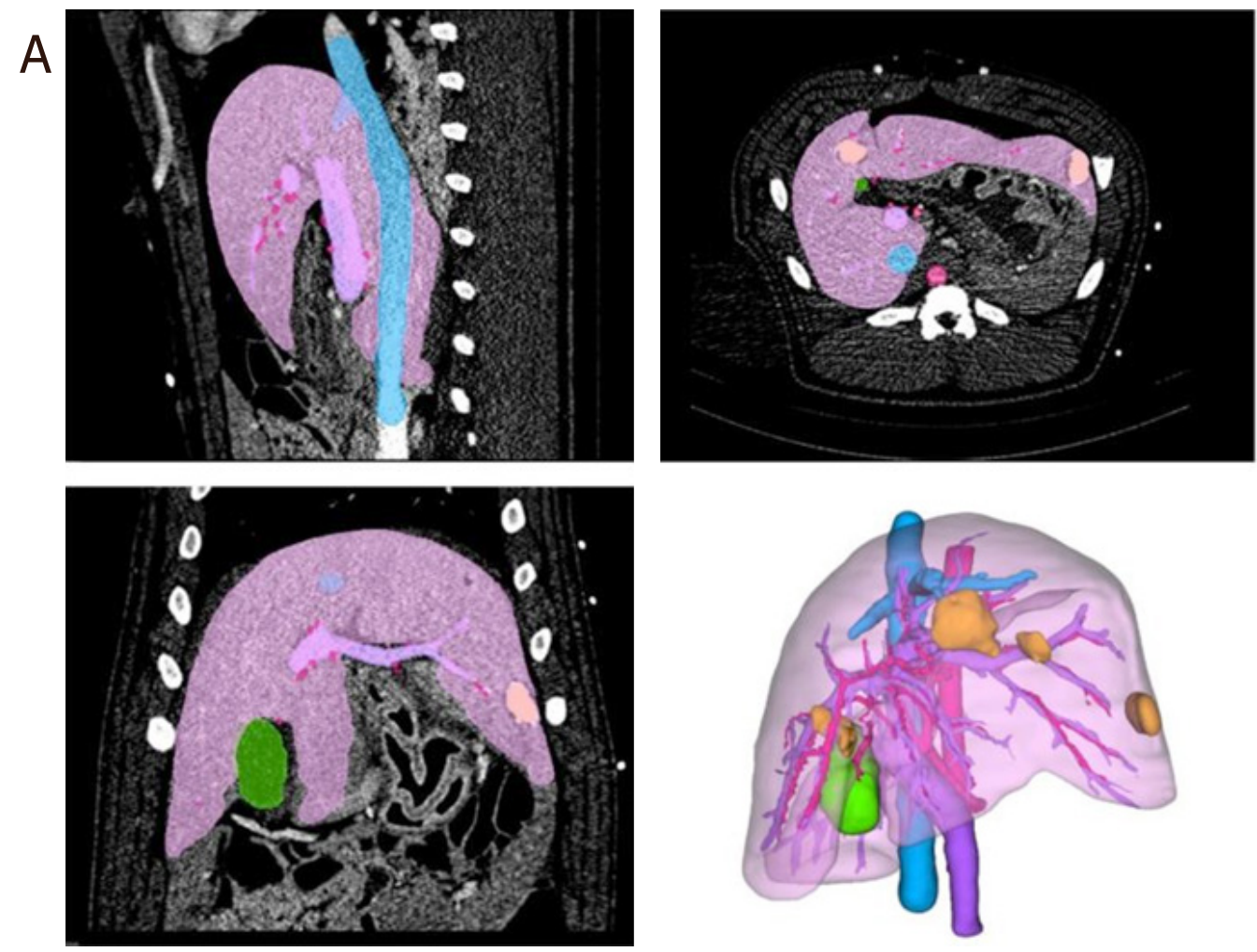

B

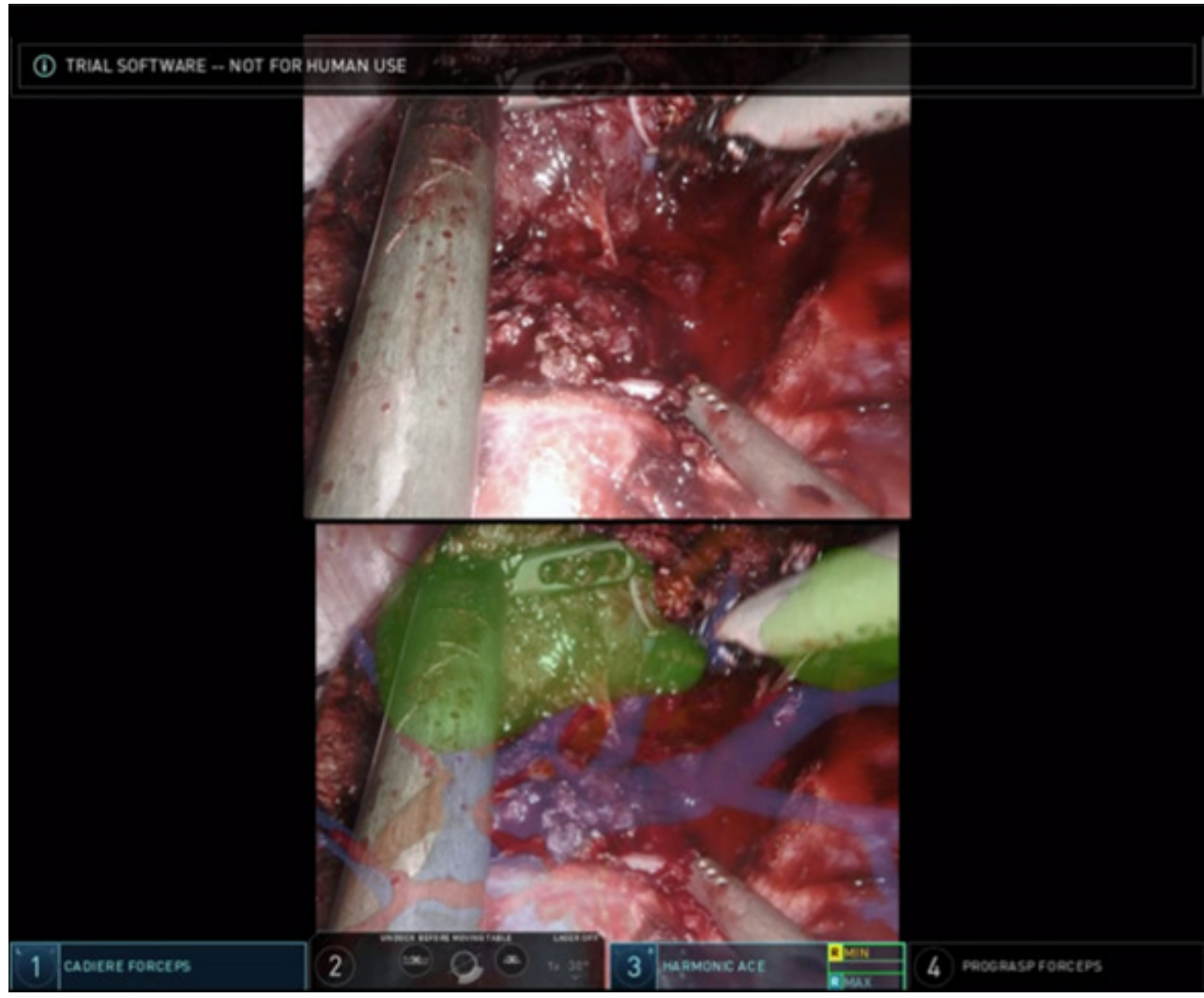

Figure 1. Real time navigation on da Vinci surgeon console. A: interactive three-dimensional anatomic representations are developed from the patient's preoperative imaging; B: 3D representations overlaid onto the surgeon's console in a porcine model to reveal the tumour's location in relation to the vascular and biliary structures in real-time. This technology can enhance the surgeon's ability to visualize the anatomy and perform a margin negative resection. Video and images courtesy of Intuitive. The da Vinci technology presented is still in development, is not $510(\mathrm{k})$ cleared and the safety and effectiveness of the product has not been established. The technology is not currently for sale in the US 
and inferior hepatic segments and may provide benefits over standard laparoscopy during resections in posteriorly and superiorly located tumours.

\section{DECLARATIONS}

\section{Authors' contributions}

Made substantial contributions to conception and design of the study and performed data analysis and interpretation: Labadie KP, Park JO, Sham JG

Performed data acquisition, as well as provided administrative, technical, and material support: Labadie KP, Park JO

\section{Availability of data and materials}

Not applicable.

\section{Financial support and sponsorship}

Dr. James O. Park received an educational grant of $<\$ 10,000$ from Intuitive Surgical Inc for research related to the $3 \mathrm{D}$ real time navigation development.

\section{Conflicts of interest}

All authors declared that there are no conflicts of interest.

\section{Ethical approval and consent to participate}

Not applicable.

\section{Consent for publication}

Not applicable.

\section{Copyright}

(c) The Author(s) 2020.

\section{REFERENCES}

1. Childers CP, Maggard-Gibbons M. Estimation of the acquisition and operating costs for robotic surgery. JAMA 2018;320:835-6.

2. Sheetz KH, Claflin J, Dimick JB. Trends in the adoption of robotic surgery for common surgical procedures. JAMA Netw Open 2020;3:e1918911.

3. Kwoh YS, Hou J, Jonckheere EA, Hayati S. A robot with improved absolute positioning accuracy for CT guided stereotactic brain surgery. IEEE Trans Biomed Eng 1988;35:153-60.

4. Satava RM. Surgical robotics: the early chronicles: a personal historical perspective. Surg Laparosc Endosc Percutan Tech 2002;12:6-16.

5. Satava RM. Emerging technologies for surgery in the 21st century. Arch Surg 1999;134:1197-202.

6. Bargar WL, Bauer A, Borner M. Primary and revision total hip replacement using the Robodoc system. Clin Orthop Relat Res 1998:82-91.

7. Alessandrini M, De Padova A, Napolitano B, Camillo A, Bruno E. The AESOP robot system for video-assisted rigid endoscopic laryngosurgery. Eur Arch Otorhinolaryngol 2008;265:1121-3.

8. Marescaux J, Leroy J, Gagner M, Rubino F, Mutter D, et al. Transatlantic robot-assisted telesurgery. Nature 2001;413:379-80.

9. Rininsland H. ARTEMIS. A telemanipulator for cardiac surgery. Eur J Cardiothorac Surg 1999;16 Suppl 2:S106-11.

10. Ballantyne GH, Moll F. The da Vinci telerobotic surgical system: the virtual operative field and telepresence surgery. Surg Clin North Am 2003;83:1293-304, vii.

11. Lanfranco AR, Castellanos AE, Desai JP, Meyers WC. Robotic surgery: a current perspective. Ann Surg 2004;239:14-21.

12. Prabhu AS, Carbonell A, Hope W, Warren J, Higgins R, et al. Robotic inguinal vs transabdominal laparoscopic inguinal hernia repair: the RIVAL randomized clinical trial. JAMA Surg 2020;155:380-7.

13. Sheetz KH, Norton EC, Dimick JB, Regenbogen SE. Perioperative outcomes and trends in the use of robotic colectomy for medicare beneficiaries from 2010 through 2016. JAMA Surg 2019;155:41-9.

14. Choi SB, Park JS, Kim JK, Hyung WJ, Kim KS, et al. Early experiences of robotic-assisted laparoscopic liver resection. Yonsei Med J 2008;49:632-8.

15. Giulianotti PC, Coratti A, Sbrana F, Addeo P, Bianco FM, et al. Robotic liver surgery: results for 70 resections. Surgery 2011;149:29-39.

16. Nota CL, Rinkes IHB, Molenaar IQ, van Santvoort HC, Fong Y, et al. Robot-assisted laparoscopic liver resection: a systematic review 
and pooled analysis of minor and major hepatectomies. HPB (Oxford) 2016;18:113-20.

17. Montalti R, Berardi G, Patriti A, Vivarelli M, Troisi RI. Outcomes of robotic vs laparoscopic hepatectomy: a systematic review and metaanalysis. World J Gastroenterol 2015;21:8441-51.

18. Kingham TP, Leung U, Kuk D, Gonen M, D’Angelica MI, et al. Robotic liver resection: a case-matched comparison. World J Surg 2016;40:1422-8.

19. Beard RE, Khan S, Troisi RI, Montalti R, Vanlander A, et al. Long-term and oncologic outcomes of robotic versus laparoscopic liver resection for metastatic colorectal cancer: a multicenter, propensity score matching analysis. World J Surg 2020;44:887-95.

20. Liu R, Wakabayashi G, Kim HJ, Choi GH, Yiengpruksawan A, et al. International consensus statement on robotic hepatectomy surgery in 2018. World J Gastroenterol 2019;25:1432-44.

21. Magistri P, Tarantino G, Assirati G, Olivieri T, Catellani B, et al. Robotic liver resection for hepatocellular carcinoma: a systematic review. Int J Med Robot 2019;15:e2004.

22. Chen PD, Wu CY, Hu RH, Chou WH, Lai HS, et al. Robotic versus open hepatectomy for hepatocellular carcinoma: a matched comparison. Ann Surg Oncol 2017;24:1021-8.

23. Pawlik TM, Scoggins CR, Zorzi D, Abdalla EK, Andres A, et al. Effect of surgical margin status on survival and site of recurrence after hepatic resection for colorectal metastases. Ann Surg 2005;241:715-22, discussion 22-4.

24. Khan S, Beard RE, Kingham PT, Fong Y, Boerner T, et al. Long-term oncologic outcomes following robotic liver resections for primary hepatobiliary malignancies: a multicenter study. Ann Surg Oncol 2018;25:2652-60.

25. Wang WH, Kuo KK, Wang SN, Lee KT. Oncological and surgical result of hepatoma after robot surgery. Surg Endosc 2018;32:3918-24.

26. Lai EC, Yang GP, Tang CN. Robot-assisted laparoscopic liver resection for hepatocellular carcinoma: short-term outcome. Am J Surg 2013;205:697-702.

27. Qiu J, Chen S, Chengyou D. A systematic review of robotic-assisted liver resection and meta-analysis of robotic versus laparoscopic hepatectomy for hepatic neoplasms. Surg Endosc 2016;30:862-75.

28. Tsung A, Geller DA, Sukato DC, Sabbaghian S, Tohme S, et al. Robotic versus laparoscopic hepatectomy: a matched comparison. Ann Surg 2014;259:549-55.

29. Magistri P, Tarantino G, Guidetti C, Assirati G, Olivieri T, et al. Laparoscopic versus robotic surgery for hepatocellular carcinoma: the first 46 consecutive cases. J Surg Res 2017;217:92-9.

30. Cho JY, Han HS, Yoon YS, Shin SH. Feasibility of laparoscopic liver resection for tumors located in the posterosuperior segments of the liver, with a special reference to overcoming current limitations on tumor location. Surgery 2008;144:32-8.

31. Teramoto K, Kawamura T, Takamatsu S, Noguchi N, Nakamura N, et al. Laparoscopic and thoracoscopic partial hepatectomy for hepatocellular carcinoma. World J Surg 2003;27:1131-6.

32. Troisi RI, Patriti A, Montalti R, Casciola L. Robot assistance in liver surgery: a real advantage over a fully laparoscopic approach? Results of a comparative bi-institutional analysis. Int J Med Robot 2013;9:160-6.

33. Casciola L, Patriti A, Ceccarelli G, Bartoli A, Ceribelli C, et al. Robot-assisted parenchymal-sparing liver surgery including lesions located in the posterosuperior segments. Surg Endosc 2011;25:3815-24.

34. Patriti A, Cipriani F, Ratti F, Bartoli A, Ceccarelli G, et al. Robot-assisted versus open liver resection in the right posterior section. JSLS 2014;18:e2014.00040.

35. Leung U, Fong Y. Robotic liver surgery. Hepatobiliary Surg Nutr 2014;3:288-94.

36. Nota CL, Woo Y, Raoof M, Boerner T, Molenaar IQ, et al. Robotic versus open minor liver resections of the posterosuperior segments: a multinational, propensity score-matched study. Ann Surg Oncol 2019;26:583-90.

37. Zygomalas A, Kehagias I. Up-to-date intraoperative computer assisted solutions for liver surgery. World J Gastrointest Surg 2019;11:1-10. 